\title{
La identidad docente: constantes y desafíos
}

\author{
Teacher identity: constant and challenges
}

\author{
Carlos Marcelo García*
}

Fecha de recepción: 8 de mayo de 2009

Fecha de revisión: 12 de junio de 2009

Fecha de aprobación: 12 de agosto de 2009

\section{Resumen}

La docencia como profesión está sufriendo cambios debidos a la evolución de las demandas de la sociedad hacia los sistemas educativos, así como por los cambios en la manera de apropiarse de lo que concede legitimidad al profesorado: el conocimiento. La cultura profesional docente, como la de casi todas las profesiones, es reacia a los cambios. Pero necesariamente hemos de evolucionar para seguir siendo una profesión imprescindible en nuestras sociedades. La identidad profesional docente se caracteriza por unas señas de identidad que intentamos revisar a lo largo de este artículo. Una profesión docente en la que la socialización previa influye en las creencias que el profesorado desarrolla, en la que el contenido que

* Docente del Departamento de Didáctica y Organización Educativa de la Facultad de Ciencias de la Educación de la Universidad de Sevilla (España). Integrante del grupo de investigación IDEA! Información disponible en: http://prometeo.us.es/idea 
se enseña crea identidad, con una clara predilección por el conocimiento práctico y el aprender de la experiencia, caracterizada por el aislamiento, y en donde los alumnos son la principal fuente de motivación del profesorado. La carrera docente, generalmente plana, también caracteriza a la profesión docente, en la que el profesorado se percibe a sí mismo como artesano, y donde está mal visto pedir ayuda y recibir retroalimentación, con poca confianza por las tecnologías, y que descuida la atención a los docentes que empiezan.

Palabras clave: profesión docente, identidad docente, desarrollo profesional, formación de profesores.

\section{Abstract}

As a profession, teaching is suffering changes as a result of the changing demands of society towards education systems, as well as for the changes in the way of appropriating of what grants legitimacy to teaching: the knowledge. The professional educational culture, like almost every profession, is reluctant to change. But necessarily we have to evolve to continue being a profession essential in our societies. The teacher's professional identity is characterized by signs of identify that we try to check throughout this article. A teaching profession in which the previous socialization influences the beliefs teachers develop, in which the content that is taught creates identity, with a clear preference for the practical knowledge and learning from experience, is characterized by the isolation, where pupils are the main source of motivation of teachers. The teaching career, generally flat, also characterizes the teaching profession, in which teachers are perceived by themselves as craftsmen, and where it is seen badly to ask for help, to receive feedback, with few confidence in technologies, and neglected attention to beginning teachers.

Key words: teaching profession, teacher's identity, professional development, teacher training. 


\section{Introducción}

Percibimos que nuestras sociedades están cambiando. Una transformación no planificada que está afectando a la forma como nos organizamos, cómo trabajamos, cómo nos relacionamos y cómo aprendemos. Estos cambios tienen un reflejo visible en la escuela como institución encargada de formar a los nuevos ciudadanos (Marcelo, 2002).

Una de las características de la sociedad en la que vivimos tiene que ver con que el conocimiento; éste es uno de los principales valores de sus ciudadanos. El valor de las sociedades actuales está directamente relacionado con el nivel de formación de sus ciudadanos, de la capacidad de innovación y de emprendimiento que estos posean. Pero los conocimientos, en nuestros días, tienen fecha de caducidad y ello nos obliga ahora más que nunca a establecer garantías formales e informales para que los ciudadanos y profesionales actualicen constantemente su competencia. Hemos entrado en una sociedad que exige de los profesionales una permanente actividad de formación y aprendizaje.

¿En qué afectan estos cambios a los docentes y a su identidad como profesionales? ¿Cómo debemos repensar el trabajo del profesor en estas nuevas circunstancias? Recientes informes internacionales han venido a centrarse y a destacar el importante papel que el profesorado juega en relación con las posibilidades de aprendizaje de los alumnos. Ya el mismo título del informe que la Organización para
Ia Cooperación y el Desarrollo Económico ([OCDE], 2005) ha publicado recientemente nos llama la atención: Teachers matter: attracting, developing and retaining effective teachers. Se afirma en el título que los profesores cuentan e importan para ayudar a mejorar la calidad de la enseñanza que reciben los alumnos. Se afirma en este informe que:

\begin{abstract}
Existe actualmente un volumen considerable de investigación que indica que la calidad de los profesores y de su enseñanza es el factor más importante para explicar los resultados de los alumnos. Existen también considerables evidencias de que los profesores varían en su eficacia. Las diferencias entre los resultados de los alumnos a veces son mayores dentro de la propia escuela que entre escuelas. La enseñanza es un trabajo exigente, y no es posible para cualquiera ser un profesor eficaz y mantener esta eficacia a lo largo del tiempo (p. 12).
\end{abstract}

Este informe viene a mostrar la preocupación internacional en relación con el profesorado, con las formas de hacer de la docencia una profesión atractiva, con cómo mantener en la enseñanza a los mejores profesores y cómo conseguir que los profesores sigan aprendiendo a lo largo de su carrera.

Este informe de la OCDE (2005) viene a mostrar que el profesorado cuenta para influir en el aprendizaje de los alumnos, para mejorar la calidad de la educación que las escuelas e institutos llevan a cabo día a día. Cuenta en definitiva como una profesión necesaria e imprescindible para la sociedad del conocimiento, y puesto que el profesorado hay que tenerlo 
en cuenta, se necesita que nuestros sistemas educativos sean capaces de atraer a los mejores candidatos para convertirse en docentes. Necesitamos buenas políticas para que la formación inicial de estos profesores les asegure las competencias que van a requerir a lo largo de su extensa, flexible y variada trayectoria profesional. De igual manera, la sociedad necesita buenos profesores cuya práctica profesional cumpla los estándares profesionales de calidad que aseguren el compromiso de respetar el derecho que los alumnos tienen de aprender.

Paralelamente al estudio de la OCDE, la prestigiosa Asociación americana de investigación educativa (AERA, por sus siglas en inglés) ha hecho público el informe que intenta resumir los resultados de la investigación sobre la formación docente, así como hacer propuestas de política educativa acordes con estos resultados. Se afirma que "en toda la nación existe un consenso emergente acerca de que el profesorado influye de manera significativa en el aprendizaje de los alumnos y en la eficacia de la escuela" (Cochran-Smith \& Fries, 2005, p. 40). En la misma línea, Darling-Hammond (2000) venía de afirmar que el aprendizaje de los alumnos "depende principalmente de lo que los profesores conocen y de lo que pueden hacer".

Pero destacar la importancia del papel del docente no es suficiente. Chapman y Aspin (2001) planteaban la necesidad de realizar profundas transformaciones en los sistemas educativos actuales para que podamos enfrentarnos a los desafíos de la sociedad del conocimiento. Estos autores plantean una serie de principios que nos parecen de interés:
- La necesidad de ofrecer oportunidades educativas que respondan a los principios de: eficacia económica, justicia social, inclusión social, participación democrática y desarrollo personal.

- La necesidad de reevaluar los currículos tradicionales y las formas de enseñar en respuesta a los desafíos educativos producidos por los cambios económicos y sociales y las tendencias asociadas al surgimiento de una economía del conocimiento y una sociedad del aprendizaje.

- La reevaluación y redefinición de los lugares donde el aprendizaje tiene lugar, así como la creación de ambientes de aprendizaje flexibles que sean positivos, estimulantes y motivadores, y que superen las limitaciones de currículo estandarizados, división por materias, limitados tiempos y rígidas pedagogías.

- Una aceptación de la importancia del valor añadido que aporta el aprendizaje. La consciencia de que aunque se empiece a entender que la escuela no sea la principal fuente de adquisición de conocimiento, se está convirtiendo en institución fundamental en la socialización de la gente joven.

- La idea de que los itinerarios de aprendizaje entre las escuelas e instituciones de educación superior, trabajadores y otros proveedores de educación tendrán un alto impacto en la formación de relaciones entre la escuela y la comunidad.

- La necesidad de promover la idea de la escuela como comunidad de aprendizaje y como centros de aprendizaje a lo largo de la vida. 
Se está demandando, por tanto, un profesor entendido como un "trabajador del conocimiento", diseñador de ambientes de aprendizaje, con capacidad para rentabilizar los diferentes espacios en donde se produce el conocimiento, y una profesión docente caracterizada por lo que Shulman (1998) ha denominado como una comunidad de práctica a través de la cual "la experiencia individual pueda convertirse en colectiva" (p. 521). Una profesión que necesita cambiar su cultura profesional, marcada por el aislamiento y las dificultades para aprender de otros y con otros; en la que está mal visto pedir ayuda o reconocer dificultades.

Por otra parte, las evidencias de los informes internacionales muestran que las políticas de reforma educativa llevadas a cabo en muchos países han deteriorado las condiciones de trabajo de los docentes, produciendo desmoralización, abandono de la profesión, absentismo, teniendo todo esto un impacto negativo en la calidad de la educación ofrecida a los alumnos. Como afirman Day, Elliott \& Kington (2005): "los profesores están dejando aparte lo que consideran como parte esencial de su trabajo, la interacción con los alumnos, para abordar las prioridades de gestión y de evaluación". Hay evidencias con relación a que los cambios en las condiciones internas y externas de las escuelas han producido condiciones de extrema incertidumbre y crisis de identidad dentro de lo que históricamente ha sido para muchos profesores una profesión estable (Day, Elliot \& Kington, 2005).

\section{Constantes en la identidad profesional docente}

Es importante considerar esta reflexión porque es a través de nuestra identidad que nos percibimos, nos vemos y queremos que nos vean. Desde el punto de vista de Lasky (2005), la identidad profesional es la forma como los profesores se definen a sí mismos y a los otros. Es una construcción del sí mismo profesional que evoluciona a lo largo de la carrera docente y que puede verse influido por la escuela, las reformas y los contextos políticos que "incluye el compromiso personal, la disposición para aprender a enseñar, las creencias, valores, conocimiento sobre la material que enseñan así como sobre la enseñanza, experiencias pasadas, así como la vulnerabilidad profesional". Las identidades profesionales configuran un "complejo entramado de historias, conocimientos, procesos y rituales" (Sloan, 2006).

Hay que entender el concepto de identidad docente como una realidad que evoluciona y se desarrolla tanto personal como colectivamente. La identidad no es algo que se posea sino algo que se desarrolla a lo largo de la vida. La identidad no es un atributo fijo para una persona, sino que es un fenómeno relacional. El desarrollo de la identidad ocurre en el terreno de lo intersubjetivo y se caracteriza como un proceso evolutivo, un proceso de interpretación de uno mismo como persona dentro de un determinado contexto. Dentro de este contexto, la identidad puede entenderse como una respuesta a la pregunta ¿quién soy en este momento? La identidad profesional no 
es una entidad estable, unitaria o fija. Resulta de un complejo y dinámico equilibrio donde la imagen propia como profesional se tiene que equilibrar con una variedad de roles que los profesores sienten que deben jugar (Beijaard, Meijer \& Verloop, 2004). Estos autores han revisado las recientes investigaciones sobre identidad profesional docente encontrando las siguientes características:

- La identidad profesional es un proceso evolutivo de interpretación y reinterpretación de experiencias, una noción que se corresponde con la idea de que el desarrollo del profesorado nunca se detiene y que se entiende como un aprendizaje a lo largo de la vida. Desde este punto de vista, la formación de la identidad profesional no es la respuesta a la pregunta ¿quién soy en este momento?, sino la respuesta a la pregunta ¿qué quiero llegar a ser?

- La identidad profesional implica tanto a la persona como al contexto. La identidad profesional no es única. Se espera que el profesorado se comporte de manera profesional, pero no porque adopte características profesionales (conocimientos y actitudes) prescritas. Los profesores se diferencian entre sí en función de la importancia que dan a estas características, desarrollando su propia respuesta al contexto.

- La identidad profesional docente está compuesta por subidentidades más o menos relacionadas entre sí. Estas subidentidades tienen que ver con los diferentes contextos en los que los profesores se mueven. Es importante que estas subidentidades no entren en conflicto. Éste aparece por ejem- plo en situaciones de cambios educativos o cambios en las condiciones de trabajo; cuanto más importante es una subidentidad, más difícil es cambiarla.

- La identidad profesional contribuye a la percepción de autoeficacia, motivación, compromiso y satisfacción en el trabajo del profesorado y es un factor importante para convertirse en un buen profesor. La identidad está influida por aspectos personales, sociales y cognitivos.

La identidad profesional docente está en estos momentos bajo revisión. En su reciente libro, Bolívar (2006) reflexiona y analiza la crisis de identidad profesional de los docentes, especialmente en el nivel de enseñanza secundaria. Desde el punto de vista de Bolívar,

las mutaciones de las últimas décadas generan sobre la situación profesional de los profesores ambigüedades y contradicciones. La crisis de identidad profesional docente ha de ser comprendida en el marco de un cierto desmoronamiento de los principios ilustrados modernos que daban sentido al sistema escolar. (Bolívar, p. 21)

Estos cambios no sólo tienen que ver con la propia profesión docente sino con "un cuadro más general de transformaciones sociales, que ha resquebrajado los espacios tradicionales de identificación sexual, religiosa, familiar o laboral" (Bolívar, 2006, p. 25). Unas transformaciones en las que lo local y lo global, la estabilidad y el cambio, están jugando un papel desestabilizador en relación con las certezas que en otras décadas han caracterizado nuestras sociedades. 
Aunque en estos momentos sea mucho más evidente, la profesión docente ha ido arrastrando a lo largo su historia un déficit de consideración social, basado, según algunos, en las características específicas de las condiciones de trabajo que la asemejan más a ocupaciones que a verdaderas profesiones como la Medicina o el Derecho. Se ha querido comparar sistemáticamente a la docencia con estas otras profesiones para ver si cumple las condiciones de

un conjunto de individuos que aplican un conocimiento científico avanzado para proporcionar un servicio a los clientes y se agrupan juntos mediante la pertenencia a un cuerpo profesional que asume la responsabilidad de controlar los promedios profesionales, y que les confiere beneficios y puede imponer sanciones a los miembros (Tomlinson, 1997).

Evidentemente, como Hoyle y John (1995) mostraban, la profesión docente, por sus especiales características, no cumple con estos estrictos y clasistas criterios.

Con el discurrir de los tiempos y la implantación de las reformas educativas, la profesión docente ha ido cambiando; para algunos, hacia una desprofesionalización, debido a la pérdida progresiva de autonomía y control interno; para otros, hacia una reprofesionalización, justificada por la necesidad de ampliar las tareas habitualmente asignadas a los docentes (Marcelo, 1999).

En el primero de los sentidos se manifestaba Hargreaves (1997), para quien las reclama- ciones y luchas actuales en el sentido de una mayor profesionalización (mayor autonomía y autocontrol interno de la profesión) de los docentes llega históricamente tarde. El avance imparable de la sociedad de la información, auspiciada por el uso de las nuevas tecnologías, va a configurar -según Hargreaves (1997) un escenario caracterizado por una "progresiva desprofesionalización: una sociedad del aprendizaje donde todo el mundo enseña y aprende y nadie es un experto" (p. 19).

Junto con el concepto de profesionalización, hemos hablado del profesionalismo, entendido en este caso como la capacidad de los individuos y de las instituciones en las que trabajan de desarrollar una actividad de calidad, comprometida con los clientes y en un ambiente de colaboración. Los estudios sobre profesionalismo han tomado en consideración la necesidad de reprofesionalizar la función docente, han percibido que la ampliación de funciones es positiva y muestran un síntoma claro de que los docentes son capaces de realizar funciones que van más allá de las tareas tradicionales, centradas en los alumnos y restringidas al espacio físico del aula. Este nuevo profesionalismo, o profesionalismo extendido, según la consideración de Hargreaves \& Goodson (1996), se concreta principalmente en las actuales demandas a los profesores para que trabajen en equipo, colaboren, planifiquen conjuntamente; pero también incluye la realización de funciones mentoras o relacionadas con la formación inicial de los profesores, así como aspectos más centrados en la formación, como la formación basada en la escuela. 
Algunos autores están llamando la atención acerca de la ironía de que, mientras se está vendiendo a los profesores y a las escuelas la idea de que deberían ser más autónomos y responsables de las necesidades propias, a la vez se les está transmitiendo cómo deben ser sus resultados y cómo deben abordar las prioridades nacionales para mejorar la competencia internacional. Se supone que los profesores están teniendo más autonomía escolar, precisamente en el mismo momento en que los parámetros con los que se espera que trabajen y mediante los cuales serán evaluados están siendo cada vez más serios y limitados (Day, 2001; Little \& McLaughlin, 1993; Smyth, 1995).

¿Cómo vemos en estos momentos la profesión docente y sus señas de identidad? ¿Qué elementos caracterizan a la identidad docente? ¿Podemos identificar dimensiones que permitan de forma constante identificar al docente y diferenciar su cultura e identidad de otros profesionales? Es algo que vamos a pretender realizar a partir de ahora estableciendo lo que creemos pueden ser esas señas de identidad que nos han venido caracterizando.

Cada una de estas constantes representa también un desafío. El desafío de desarrollar procesos que ayuden a situar a la profesión docente como una "profesión del conocimiento", comprometida con el derecho de aprender de los alumnos. No se trata por lo tanto de esperar a que los cambios llamen a la puerta de la escuela. No se trata tampoco de introducir computadores en las aulas como signo externo de ultramodernidad. La docencia como profesión necesita revisarse y reconstruirse para seguir cumpliendo los compromisos morales que ha venido desarrollando: asegurar el derecho de aprender de todos: niños y niñas, adultos y adultas.

He identificado catorce constantes. Quizás podrían ser más. Quizás podrían agruparse. Pero son lo suficientemente sugerentes como para desarrollar el debate que espero propicie esta intervención.

\section{Miles de horas como alumnos no son gratuitas: la socialización previa}

Podemos afirmar sin riesgo a equivocarnos que la docencia es la única de las profesiones en las que los futuros profesionales se ven expuestos a un mayor periodo de observación no dirigida en relación con las funciones y tareas que desempeñarán en el futuro. Como comenta Tardif (2004), la docencia

\footnotetext{
También exige una socialización en la profesión y una vivencia profesional a través de las cuales la identidad profesional va siendo poco a poco construida y experimentada $y$ en donde entran en juego elementos emocionales, de relación y simbólicos que permiten que un individuo se considere y viva como profesor y asuma así, subjetiva y objetivamente, el hecho de realizar una carrera en la enseñanza (p. 79).
}

Por otra parte, ya resulta clásico el trabajo que realizó Lortie (1975) en relación con la socialización previa en la enseñanza. Este autor encontró que los profesores desarrollan patrones mentales, creencias sobre la enseñanza a partir del periodo tan prolongado de observación como alumnos. 
La identidad docente va configurándose así, de forma paulatina y poco reflexiva, a través de lo que podríamos denominar aprendizaje informal y mediante la observación en futuros docentes que van recibiendo modelos docentes con los que se van identificando poco a poco y en cuya identificación influyen más aspectos emocionales que racionales. $Y$ como comentan Van Veen, Sleegers y Van den Ven (2005):

Dado que la interacción humana es tan importante en la práctica docente y que los profesores a menudo se implican muy profundamente en su trabajo, las recientes investigaciones vienen a afirmar que las emociones constituyen un elemento esencial en el trabajo y la identidad de los profesores (p. 918).

Ese vínculo entre los aspectos emocionales y cognitivos de la identidad profesional docente han de ser tenidos en cuenta a la hora de plantear propuestas formativas, ya que, como veremos a continuación, las emociones configuran una parte muy importante de las creencias que los profesores desarrollamos acerca de cómo se enseña, se aprende y se aprende a enseñar. Cambiar estas creencias requiere también una fuerte implicación emocional.

\section{Las creencias sobre la enseñanza dirigen la práctica profesional}

Como hemos comentado anteriormente, los aspirantes a profesores no son "vasos vacíos" cuando acceden a una institución de formación inicial docente. Tiene ya ideas y creencias fuertemente asentadas sobre qué es enseñar y aprender. En la investigación sobre la formación inicial ha habido una especial preocupación por el análisis de las creencias que los profesores en formación traen cuando inician su andadura profesional. Se ha entendido que las creencias son como proposiciones, premisas que mantienen las personas acerca de lo que consideran verdadero. Las creencias, a diferencia del conocimiento proposicional, no requieren una condición de verdad contrastada y cumplen dos funciones en el proceso de aprender a enseñar. En primer lugar, las creencias influyen en la forma como aprenden los profesores y, en segundo lugar, las creencias influyen en los procesos de cambio que los profesores puedan intentar (Richardson, 1996).

La literatura de investigación sobre aprender a enseñar ha identificado tres categorías de experiencias que influyen en las creencias y conocimientos que los profesores desarrollan sobre la enseñanza:

1. Experiencias personales. Incluyen aspectos de la vida que determinan una visión del mundo, creencias hacia uno mismo y en relación con los demás, ideas acerca de las relaciones entre la escuela y la sociedad, así como sobre la familia y la cultura; la procedencia socioeconómica, étnica, el sexo, religión pueden afectar las creencias acerca del aprender a enseñar.

2. Experiencia con el conocimiento formal. El conocimiento formal, entendido como aquello sobre lo que debe trabajarse en la escuela. Las creencias acerca de la materia que se enseña así como la forma de enseñarla. 
3. Experiencia escolar y de aula. Incluye todas aquellas experiencias como estudiante que contribuyen a formar una idea acerca de qué es enseñar y cuál es el trabajo del profesor.

De entre los hallazgos más divulgados está el hecho de que las creencias que los profesores en formación traen consigo cuando inician su formación inicial afectan de una manera directa a la interpretación y valoración que los profesores hacen de las experiencias de formación del profesorado. Esta modalidad de aprender a enseñar se produce a través de lo que se ha denominado aprendizaje por la observación (Lortie, 1975), aprendizaje que en muchas ocasiones no se genera de manera intencionada sino que se va adentrando en las estructuras cognitivas -y emocionales- de los futuros profesores, de manera inconsciente, llegando a crear expectativas y creencias difíciles de remover.

Hay que hacer referencia a Pajares (1992) como uno de los investigadores que ha realizado más contribuciones en relación con el análisis de las creencias. Llamó la atención respecto a la dispersión semántica que ha caracterizado a esta línea de investigaciones, en las que se han utilizado términos como creencia, actitud, valores, juicios, axiomas, opiniones, ideología, percepciones, concepciones, sistema conceptual, preconcepciones, disposiciones, teorías implícitas, teorías explícitas, teorías personales, procesos mentales internos, reglas de la práctica, principios prácticos, etc. Esta dispersión semántica ha producido que los resultados de investigaciones no puedan compararse por no compartir un mismo marco conceptual. Pajares ha diferenciado entre conocimiento y creencias, poniendo de manifiesto que las creencias, a diferencia del conocimiento, poseen una clara connotación afectiva y evaluativa: "el conocimiento de un tema se diferencia de los sentimientos que tengamos sobre ese tema, de la misma manera que se diferencia entre autoconcepto y autoestima, entre conocimiento de sí mismo y sentimiento del valor propio" (Pajares, 1992).

Desde esta diferenciación, las investigaciones han venido mostrando que los profesores en formación entran en el programa de formación con creencias personales acerca de la enseñanza, con imágenes de buen profesor, imagen de sí mismos como profesores y la memoria de sí mismos como alumnos. Estas creencias e imágenes personales generalmente permanecen sin cambios a lo largo del programa de formación y acompaña a los profesores durante sus prácticas de enseñanza (Feiman-Nemser, 2001; Kagan, 1992; Wideen, Mayer-Smith \& Moon, 1998). En esta línea, afirma FeimanNemser (2001, p. 1016) que las imágenes y creencias que los profesores en formación traen consigo cuando inician su formación inicial actúan como filtros mediante los cuales dan sentido a los conocimientos y experiencias con los que se encuentran. Pero también pueden actuar como barreras al cambio limitando las ideas que los alumnos pueden llegar a desarrollar sobre la enseñanza.

Pajares (1992) sintetizó los resultados de la investigación de las creencias de los profesores en los siguientes principios: 
- Las creencias se forman en edad temprana y tienden a perpetuarse, superando contradicciones causadas por la razón, el tiempo, la escuela o la experiencia.

- Los individuos desarrollan un sistema de creencias que estructura todas las creencias adquiridas a lo largo del proceso de transmisión cultural.

- Los sistemas de creencias tienen una función adaptativa al ayudar al individuo a definir y comprender el mundo y a sí mismos.

Conocimiento y creencias están interrelacionados, pero el carácter afectivo, evaluativo y episódico de las creencias se convierten en un filtro a través del cual todo nuevo fenómeno se interpreta. Las subestructuras de creencias, como son las creencias educativas, se deben comprender en términos de sus conexiones con las demás creencias del sistema.

Debido a su naturaleza y origen, algunas creencias son más indiscutibles que otras. Cuanto más antigua sea una creencia, más difícil es cambiarla. Las nuevas creencias son más vulnerables al cambio. El cambio de creencias en los adultos es un fenómeno muy raro. Los individuos tienden a mantener creencias basadas en conocimiento incompleto o incorrecto.

Las creencias son instrumentales al definir tareas y al seleccionar los instrumentos cognitivos con los cuales interpretar, planificar y tomar decisiones con relación a estas tareas; por lo tanto juegan un papel crucial al definir la conducta y organizar el conocimiento y la información.
Pero al igual que desarrollamos conocimientos y creencias generales acerca de la enseñanza, los alumnos, la escuela o el profesor, la materia que enseñamos o pretendemos enseñar no se queda al margen de nuestras concepciones. La forma como conocemos una determinada disciplina o área curricular afecta la forma cómo la enseñamos. Existen múltiples evidencias que nos muestran ciertos arquetipos que los profesores en formación tienen sobre la disciplina que estudian, ya sea ésta matemáticas, lengua o educación física. Preguntas como ¿qué son y para qué sirven las matemáticas, la lengua, la educación física, etc.?, son necesarias de plantear cuando pretendemos "partir de lo que el alumno ya sabe". Tomando el contenido que se enseña y se aprende como argumento de la indagación, podemos encontrar diferencias en el comportamiento observable de profesores en función del dominio que posean del contenido que enseñan (Onofre, 2000).

Una de las claves de identidad profesional docente la proporciona sin lugar a dudas el contenido que se enseña. Esto es especialmente cierto conforme avanzamos en el nivel educativo: menor en educación infantil y mayor en enseñanza secundaria y universitaria.

Resulta de todo punto de vista importante -y de ahí su influencia en la construcción de la identidad profesional docente- un conocimiento profundo del contenido que se enseña. Al respecto, Buchmann (1984) nos señala que "conocer algo nos permite enseñarlo; y conocer un contenido con profundidad significa estar mentalmente 
organizado y bien preparado para enseñarlo de una forma general" (p. 34). Cuando el docente no posee conocimientos adecuados de la estructura de la disciplina que está enseñando, puede representar erróneamente el contenido a los alumnos. El conocimiento que los profesores poseen del contenido a enseñar también influye en el qué y el cómo enseñar.

El conocimiento del contenido incluye diferentes componentes, de los cuales dos son los más representativos: conocimiento sintáctico y sustantivo. El conocimiento sustantivo se constituye con la información, las ideas y los tópicos por conocer, es decir, el cuerpo de conocimientos generales de una materia, los conceptos específicos, las definiciones, las convenciones y los procedimientos. Este conocimiento es importante en la medida en que determina lo que los profesores van a enseñar y desde qué perspectiva lo harán. Por ejemplo, en Historia, el marco de análisis cultural, político o ideológico que se escoja, puede determinar el qué se enseña y cómo se enseña. El conocimiento sintáctico del contenido completa al anterior y se encarna en el dominio que tiene el formador de los paradigmas de investigación en cada disciplina, del conocimiento en relación con cuestiones como la validez, las tendencias, las perspectivas y la investigación en el campo de su especialidad. En Historia, incluiría las diferentes perspectivas de interpretación de un mismo fenómeno; en Ciencias Naturales, el conocimiento sobre el empirismo y el método de investigación científica, etc.
Un trabajo acerca de este tema (GessNewsome, 2003) indica que el conocimiento del contenido de la mayoría de los profesores principiantes es fragmentado y pobremente organizado, lo cual dificulta el acceso eficiente a este conocimiento mientras se está enseñando. Como consecuencia, muchos profesores principiantes se ven forzados a enseñar algoritmos y hechos que recuerdan de sus años escolares. Un bajo nivel de conocimientos pobremente organizados influye en la enseñanza de varias formas. Cuando los profesores principiantes planifican, generalmente se basan en los libros de texto como punto de partida para la enseñanza. Enseñar se equipara a recordar información. Este conocimiento superficial perjudica a los alumnos al limitarles una comprensión de los conceptos y al llevarles a representaciones erróneas de la disciplina. Los profesores principiantes formulan preguntas de bajo nivel y limitan las actividades de los alumnos a aspectos estrictamente procedimentales. Los profesores principiantes son incapaces de conectar los comentarios y preguntas de los alumnos con otros temas y a menudo rechazan respuestas alternativas de los alumnos. El resultado es la gestión del trabajo de los alumnos en lugar de controlar su comprensión. Los profesores con un conocimiento conceptual profundo de la material realizan más conexiones y relaciones con otros tópicos y pueden trasladar este conocimiento a la enseñanza y a la resolución de problemas. 


\section{Fragmentación del conocimiento docente: algunos conocimientos valen más que otros}

Según lo que podríamos denominar la sabiduría popular, para enseñar basta con saber la materia que se enseña. El conocimiento del contenido parece que es una seña de identidad y reconocimiento social. Pero para enseñar sabemos que el conocimiento de la materia no es un indicador de calidad de enseñanza. Existen otros tipos de conocimientos también importantes: conocimiento del contexto (dónde se enseña), de los alumnos (a quién se enseña), de sí mismo y también de cómo se enseña.

De esta forma, el conocimiento didáctico del contenido aparece como un elemento central de los saberes del docente; representa la combinación adecuada entre el conocimiento de la materia a enseñar y el conocimiento pedagógico y didáctico referido a cómo enseñarla. En los últimos años, se ha venido trabajando en diferentes contextos educativos para clarificar cuáles son los componentes de este tipo de conocimiento profesional de la enseñanza. El conocimiento didáctico del contenido, como línea de investigación, representa la confluencia de esfuerzos de investigadores didácticos con investigadores de materias específicas preocupados por la formación del profesorado. El conocimiento didáctico del contenido nos dirige a un debate en relación con la forma de organización y de representación del conocimiento, a través de analogías y metáforas. Plantea la necesidad de que los profesores en formación adquieran un conocimiento experto del contenido por enseñar, para que puedan desarrollar una enseñanza que propicie la comprensión de los alumnos.
Shulman (1992) manifestaba la necesidad de que los profesores construyeran puentes entre el significado del contenido curricular y la construcción realizada por los alumnos de ese significado. Este prestigioso investigador afirma:

Los profesores llevan a cabo esta hazaña de honestidad intelectual mediante una comprensión profunda, flexible y abierta del contenido; comprendiendo las dificultades más probables que tendrán los alumnos con estas ideas...; comprendiendo las variaciones de los métodos y modelos de enseñanza para ayudar a los alumnos en su construcción del conocimiento; y estando abierto a revisar sus objetivos, planes y procedimientos en la medida en que se desarrolla la interacción con los estudiantes. Este tipo de comprensión no es exclusivamente técnica, ni solamente reflexiva. No es sólo el conocimiento del contenido, ni el dominio genérico de métodos de enseñanza. Es una mezcla de todo lo anterior, y es principalmente pedagógico (Shulman, 1992, p. 12).

El conocimiento didáctico del contenido tiene que ver con la forma como los profesores piensan que hay que ayudar a los alumnos a comprender un determinado contenido. Incluye las formas de representar y formular el contenido para hacerlo comprensible a los demás, así como un conocimiento sobre lo que hace fácil o difícil aprender: concepciones y preconcepciones que los alumnos de diferentes edades y procedencias traen con ellos acerca de los contenidos que aprenden (Borko \& Putnam, 1996). De acuerdo con Magnusson, Krajcik y Borko (2003), el conocimiento didáctico del contenido incluye la forma de organizar 
los contenidos, los problemas que surgen, la adaptación a los alumnos con diversidad de intereses y habilidades.

Pues bien, si revisamos las mallas curriculares de los programas de formación docente, encontramos una clara fragmentación y descoordinación entre los diferentes tipos de conocimiento a los que anteriormente nos hemos referido. Por una parte, se presentan los contenidos disciplinares y, por otra, los pedagógicos. La identidad profesional docente se refuerza en relación con los contenidos que se enseñan cuando el currículo de la formación inicial así lo muestra con clara evidencia.

Esta descoordinación entre distintos tipos de conocimientos también se ve reflejada en la yuxtaposición entre universidad y escuela. Ya Feiman-Nemser (2001) y Buchmann (1984) llamaron la atención en relación con este hecho. Se referían al divorcio que existe en la formación inicial según la cual suele ocurrir que los estudiantes perciben que tanto los conocimientos como las normas de actuación en la institución de formación tienen poco que ver con los conocimientos y prácticas profesionales. En este caso los estudiantes suelen deslumbrarse por la realidad y cuando se reincorporan de nuevo a la actividad académica, comienzan a desechar, por considerarla menos importante, la necesidad de ciertos conocimientos que fundamenten el trabajo práctico.

\section{Se aprende a enseñar enseñando: el valor del conocimiento práctico}

¿Quién no ha oído más de una vez esta expresión? La práctica hace al docente mucho más que la teoría adquirida en la formación inicial. Desde esta perspectiva, se da un valor mítico a la experiencia como fuente de conocimiento sobre la enseñanza y sobre el aprender a enseñar. Zeichner (1980) utilizaba la palabra mito para referirse a la creencia según la cual "las experiencias prácticas en colegios contribuye necesariamente a formar mejores profesores. Se asume que algún tiempo de práctica es mejor que ninguno, y que cuanto más tiempo se dedique a las experiencias prácticas mejor será (p. 45).

No es posible realizar el más mínimo planteamiento conceptual sobre el valor de la experiencia en la enseñanza y en la formación del profesorado sin referirse a Dewey. En 1938, este autor defendía la necesidad de desarrollar una teoría de la experiencia, puesto que ya entonces constataba que experiencia no es sinónimo de educación. En este sentido, planteaba que "no es suficiente con insistir en la necesidad de la experiencia, ni incluso de la actividad en la experiencia. Todo depende de la calidad de la experiencia que se tenga" (Dewey, 1938, p. 27). Valorar la calidad de las experiencias supone tener en cuenta dos aspectos: un aspecto inmediato, referido a cuanto de agradable o desagradable resulta para el sujeto que la vive. El segundo aspecto tiene una mayor importancia para el tema que nos ocupa: el efecto que dicha experiencia tiene en experiencias posteriores, es decir, la transferencia para posteriores aprendizajes. 
Este reconocimiento implícito del valor que la práctica y la experiencia tienen para la formación inicial docente viene a contrastar con la primacía explícita de lo que denominaríamos conocimiento proposicional. Cochran-Smith y Lytle (1999) han reflexionado sobre las relaciones entre conocimiento y práctica en la formación del profesorado y nos plantean que las cosas pueden tener diferentes puntos de vista. Así, diferencian entre: conocimiento para la práctica, el cual entiende que la relación entre conocimiento y práctica es aquella en que el conocimiento sirve para organizar la práctica, y por ello, conocer más (contenidos, teorías educativas, estrategias instruccionales, etc.) conduce de forma más o menos directa a una práctica más eficaz, y conocimiento para enseñar, que es un conocimiento formal, derivado de la investigación universitaria, y es al que se refieren los teóricos cuando se habla de que la enseñanza ha generado un cuerpo de conocimiento diferente al conocimiento común. La práctica, desde esta perspectiva, tiene mucho que ver con la aplicación del conocimiento formal a las situaciones prácticas.

Por otra parte, el conocimiento en la práctica pone el énfasis de la investigación sobre aprender a enseñar; esto ha sido la búsqueda del conocimiento en la acción. Se ha estimado que lo que los profesores conocen está implícito en la práctica, en la reflexión sobre la práctica, en la indagación práctica y en la narrativa de esa práctica. Un supuesto de esta tendencia es que la enseñanza es una actividad incierta y espontánea, contextualizada y construida en respuesta a las particularidades de la vida diaria en las escuelas y las clases. El conocimiento está situado en la acción, en las decisiones y juicios que toman los profesores. Este conocimiento se adquiere mediante la experiencia y la deliberación y los profesores aprenden cuando tienen oportunidad de reflexionar sobre lo que hacen.

Por último, el conocimiento de la práctica se incluye dentro de la línea de investigación cualitativa, pero cercana al movimiento denominado del profesor como investigador. La idea de la que parte es que en la enseñanza no tiene sentido hablar de un conocimiento formal y otro conocimiento práctico sino que el conocimiento se construye colectivamente dentro de comunidades locales, formadas por profesores trabajando en proyectos de desarrollo de la escuela, de formación o de indagación colaborativa (Cochran-Smith y Lytle, 1999).

De estos tipos de conocimientos, podríamos afirmar que el que mejor identifica a la profesión docente es el segundo tipo de conocimiento. Un conocimiento específico del contexto, difícil de codificar ya que se expresa ligado a la acción, también moral y emocional, privado o interpersonal, comunicado de manera oral, práctico, orientado a soluciones, que se expresa en forma metafórica, narrativa, y en general, con bajo estatus y prestigio. Este tipo de conocimiento es el que Schön (1983) denominó epistemología de la práctica.

\section{El aislamiento: cada cual es dueño en su aula}

A la tarea de enseñar, los profesores siguen enfrentándose generalmente en solitario. Sólo los 
alumnos son testigos de la actuación profesional de los profesores. Pocas profesiones se caracterizan por una mayor soledad y aislamiento. A diferencia de otras profesiones u oficios, la enseñanza es una actividad que se desarrolla en solitario. Como de forma acertada afirma Bullough (1998), la clase es el santuario de los profesores: "el santuario de la clase es un elemento central de la cultura de la enseñanza, que se preserva y protege mediante el aislamiento, y que padres, directores y otros profesores dudan en violar". Cuando estamos asistiendo a propuestas que evidentemente plantean la necesidad de que los profesores colaboren, trabajen conjuntamente, etc., nos encontramos con la pertinaz realidad de profesores que se refugian en la soledad de sus clases. Ya resulta clásico el estudio llevado a cabo por Lortie (1975), en el que mediante entrevistas estableció algunas características de la profesión docente en Estados Unidos, que no sólo son de gran actualidad, sino que son perfectamente aplicables a nuestro país. Una característica identificada por Lortie fue el individualismo. Este autor comentaba que:

La forma celular de la organización escolar y la ecología de distribución del espacio y del tiempo ponen a las interacciones entre los profesores al margen de su trabajo diario. El individualismo caracteriza su socialización; los profesores no comparten una potente cultura técnica. Las mayores recompensas psíquicas de los profesores se obtienen en aislamiento de sus compañeros, y se cuidan mucho de no franquear las barreras de las clases (p. 5).

El aislamiento de los profesores está favorecido evidentemente por la arquitectura escolar que organiza la escuela en módulos estándar, así como por la distribución del tiempo y el espacio y la existencia de normas de independencia y privacidad entre los profesores. El aislamiento, como norma y cultura profesional, tiene ciertas ventajas y algunos evidentes inconvenientes para los profesores. En este sentido, señalaban Bird \& Little (1986) que, aunque el aislamiento facilita la creatividad individual y libera a los profesores de algunas de las dificultades asociadas con el trabajo compartido, también les priva de la estimulación del trabajo con los compañeros y se deja de recibir el apoyo necesario para progresar a lo largo de la carrera. Es más, cuando en el mundo de las empresas se está hablando de la necesidad de gestionar el conocimiento como medio para rentabilizar ese saber hacer que los empleados han ido acumulando a lo largo del tiempo, en la enseñanza, en palabras de Hargreaves (1999), los profesores

Ignoran el conocimiento que existe entre ellos; por tanto, no pueden compartir y construir sobre este conocimiento. Al mismo tiempo tampoco conocen el conocimiento que no poseen y por tanto no pueden generar nuevo conocimiento. Hay una compleja distribución social del conocimiento en la escuela: ningún profesor en particular conoce o puede conocer la totalidad del conocimiento profesional que los profesores poseen (p. 124).

Ello es debido a que gran parte del conocimiento de los profesores es tácito, difícil de articular y el objetivo de la gestión del conocimiento consiste en ayudar a la organización a utilizar su propio capital intelectual. 


\section{Los alumnos y la motivación profesional}

Hemos comentado anteriormente que una de las características de la profesión docente es el aislamiento. Los docentes en general desarrollan su actividad profesional con los alumnos como únicos testigos. Pero es que, como también indicara Lortie (1975), el tipo de motivación profesional docente está ligado a los alumnos. La motivación para enseñar y para seguir enseñando es una motivación intrínseca, ligada fuertemente a la satisfacción por conseguir que los alumnos aprendan, desarrollen capacidades, evolucionen, crezcan. Otras fuentes de motivación profesional, como incrementos salariales, premios, reconocimientos, están bien pero siempre en la medida en que repercutan en la mejora de la relación con el alumnado.

Esta identificación tan intensa del profesorado con los alumnos hace que algunas innovaciones derivadas de ciertas reformas educativas, que llevan a reducir tiempos de docencia directa con los alumnos para permitir tiempos de trabajo en equipo docente, de colaboración, se perciban por algunos profesores como tiempo que les están quitando a la dedicación a sus alumnos.

Pero si los alumnos juegan un papel importante en la configuración de la identidad profesional docente, no es menos ciertos que los alumnos de hoy en día han cambiado respecto a los de hace algunas décadas. Los denominados "nativos digitales", jóvenes que han nacido en la era de la computación, familiarizados con los celulares y la comunicación sincrónica, habituados a desenvolverse con comodidad en el hipertexto, amantes de los videojuegos y con capacidad de procesamiento flexible de múltiples fuentes de información, han empezado a poblar nuestras escuelas y centros de enseñanza. Esos cambios deben de ser tenidos en cuenta por los docentes para saber a qué tipo de alumnos nos estamos dirigiendo. Esta característica de la profesión docente conduce a que las señas de identidad del profesorado se ciñan mucho más al aula que a la institución en la que laboran. Esta realidad dificulta en gran medida el desarrollo de propuestas organizativas que supongan un cambio en esa cultura tan arraigada en la docencia.

\section{Carrera docente: el que sale del aula no vuelve}

Aunque en algunos países de la región las cosas están cambiando, lo cierto es que en general podemos afirmar que la carrera docente puede caracterizarse como plana. Como comentara Terigi (2007):

\footnotetext{
Históricamente el trabajo docente se configuró en la región según un modelo basado en la carrera, que sólo permite que el docente ascienda a puestos de trabajo que lo alejan del aula, y en el que el régimen de compensaciones se encuentra desvinculado de las actividades desarrolladas en las escuelas.
}

La carrera docente se entiende como un trayecto individual, poco ligado al desarrollo de actividades colectivas y cuyos resultados producen que el crecimiento en la carrera generalmente produzca un alejamiento del aula. 
La asunción por parte del profesorado de roles diferentes, como pueden ser el de supervisor, asesor o formador en general, se desarrollan fuera del aula y no se permite la compatibilidad con actividades docentes y ocurre que el que sale del aula en general no suele volver a ella.

\section{Todo depende del profesor: los docentes como artesanos}

Coherente con la característica del aislamiento docente, existe también la idea de que los profesores son responsables de todo lo que ocurre en su aula. Existe una especie de hiperresponsabilización del docente como si tanto las condiciones de acceso de los alumnos como las propias condiciones en que se desarrolla su actividad profesional no estuvieran mediatizadas por directrices, normativas y relaciones de poder así como en la escuela o en la sociedad. Por otra parte, a partir de los trabajos de Michael Huberman, se ha venido desarrollando la imagen del docente como artesano independiente. Es ésta una visión de la identidad profesional docente según la cual los profesores actúan como artesanos, construyendo conocimiento y habilidades y materiales de la misma forma que los artesanos lo realizan. Los profesores así trabajan solos en clase y acumulan sabiduría y saber hacer. El aprendizaje es autodirigido y, principalmente. Conservador, en el sentido de que el profesor conserva lo que le funciona (Sykes, 1999).

El propio Huberman (1993) afirmaba la necesidad de

legitimar el modelo del profesor como artesano, aquél que es muy individualista y sensible al contexto, y que como resultado implica la acumulación ideosincrática de un tipo de conocimiento base y de un repertorio de habilidades... Dicho claramente, estos profesores trabajan solos, aprenden solos y desarrollan la mayor parte de su satisfacción profesional solos o por sus interacciones con los alumnos en lugar de con los compañeros (pp. 22-23).

¿Esta identidad artesanal del docente impide el desarrollo de propuestas colaborativas? Desde el punto de vista de Talbert y McLaughlin (2002), puede funcionar la idea de comunidades artesanales, es decir, grupos de profesores que de forma colaborativa desarrollan soluciones, así como conocimiento, trabajando con sus propios medios y recursos.

\section{El docente como consumidor: fast-food en las aulas}

Otra visión de la docencia, sobre todo promovida desde las instancias políticas encargadas de planificar y regular la educación, es la del docente como consumidor, consumidor de propuestas de reformas diseñadas por las élites nacionales -o importadas de otros países o regiones-. A lo largo de los años noventa y en la década actual se están promoviendo reformas en los sistemas educativos, tanto en su estructura como en su contenido, que en general se caracterizan por estar diseñadas por administraciones convencidas de que la bondad de las reformas llevará innegablemente a su implantación. Así se traslada una visión del docente como un aplicador de innovaciones que a veces no entiende y en las que, por supuesto, no ha participado. 
Esta visión del docente y de los procesos de cambio en las escuelas viene a desconocer y a veces a despreciar la realidad de la cultura y la práctica profesional docente, además se piensa que los cambios en educación son procesos lineales que se implantan de forma sencilla con tal que sepamos explicar bien a los docentes en qué consisten. Sin embargo, los procesos de cambio, tanto en los individuos como en las organizaciones, no funcionan de manera racional. Los sistemas complejos generan sus propios procesos de autorregulación para acomodarse o modificar las propuestas de cambio que no siempre tienen los resultados que se prevén desde las instancias promotoras (López y Sánchez, 2000).

Por eso decimos que la idea del docente consumidor de fast-food fracasa cuando se pretende modificar por medios rápidos elementos estructurales de su identidad profesional. Hace unos años llamaba la atención sobre la progresiva burocratización e intensificación de la docencia (Marcelo, 1996). El avance de procesos de control sobre la docencia, externos al control profesional (control del mercado, control político y administrativo) viene a poner de manifiesto una imagen profesional débil. Pero también se fracasa cuando se pretende actualizar al profesorado, es decir, implicarles en actividades que promuevan su aprendizaje continuo cuando las modalidades que se ofrecen no van más allá de cursos cortos, descontextualizados, alejados de los problemas concretos y sin aplicación práctica, ni seguimiento.

\section{La competencia no reconocida y la incompetencia ignorada}

Fueron Fullan \& Hargreaves (1992) quienes acuñaron esta expresión. De alguna manera ya hemos hecho referencia al hecho de que el aislamiento y la imagen del profesor como artesano conducen a una realidad que por más que nos sorprenda sigue estando viva: los docentes son profesionales que a lo largo de su carrera profesional rara vez observan a otro docente realizando su actividad profesional, es decir, enseñando, y esta falta de hábito analítico conduce a un estancamiento profesional evidente: si no revisamos lo que hacemos, si no lo sometemos a juicio, no avanzamos, y así se producen las dos situaciones que Fullan y Hargreaves denuncian.

Por una parte, reconocemos que existen multitud de docentes, buenos docentes, que realizan su trabajo de manera honesta y profesional, implicándose y consiguiendo un buen aprendizaje de sus alumnos, desarrollando -a menudo de manera artesanal- innovaciones cuyos resultados no sobrepasan las paredes de la escuela o a lo más de la comunidad próxima. Por otra parte, la incompetencia ignorada. Existen también docentes que se refugian en el anonimato (aislamiento) característico de la identidad profesional docente para desarrollar una enseñanza que viene a defraudar claramente el derecho de aprender de los alumnos.

¿Cómo salir de este callejón o dilema? Algunos países están optando por el desarrollo de un sistema de estándares que venga a determinar con claridad cuáles son las competencias que 
debe de poseer y demostrar todo docente. En este sentido, y como han mostrado Yinger \& Hendricks (2000), los estándares educativos constituyen una de las herramientas más poderosas para la profesionalización de la enseñanza, ya que juegan un doble papel en el desarrollo de la profesión. En primer lugar, los estándares se constituyen en una buena manera de demostrar al público y a los políticos que la profesión tiene suficientes procesos de control de calidad, que hacen seguimiento del acceso y del ejercicio de una práctica eficaz, para así ganar en legitimidad social. En segundo lugar, los estándares funcionan como parámetros y orientaciones para el desarrollo del trabajo profesional, ya que pueden definir una práctica eficaz en términos de los resultados deseados. De esta manera, los estándares se convierten en la base para organizar la formación inicial y continua de los profesores, algo a lo que volveremos más adelante.

En la misma línea se ha venido mostrando Darling-Hammond (2000), para quien resulta fundamental que la enseñanza desarrolle su propio modelo de profesionalidad, un modelo de profesionalidad que no pasa necesariamente por seguir el llevado a cabo por otras profesionales que han alcanzado el reconocimiento y prestigio a través de un distanciamiento respecto de los clientes, así como de un conocimiento altamente especializado. A diferencia de este modelo, la enseñanza debe insertarse en la comunidad y conectar con la vida de los alumnos, construyendo comunidades de aprendizaje relevantes y adecuadas para responder a las necesidades e intereses de los alumnos como ciudadanos con derecho a aprender.
Y para caminar hacia ese reconocimiento, entiende que "la palanca política más importante de que se dispone para mejorar el proceso de enseñanza y aprendizaje es, probablemente, el desarrollo de unos estándares profesionales que contemplen los aspectos más importantes de la profesión docente" (Darling-Hammond, 2001). Estos estándares públicos, asumidos y desarrollados por la propia profesión, pueden ser el eje vertebrador de los programas de formación inicial y continua. Unos programas que han estado organizados sin tener en cuenta el trabajo global del profesor en la escuela y olvidando que la formación va dirigida a que los profesores aseguren el aprendizaje de los alumnos, último criterio de calidad de la formación.

\section{¿Qué hacemos con estos cacharros? Desconfianza ante las tecnologías}

Hablábamos antes de un aspecto muy característico de la identidad profesional docente por el que, siguiendo a Fullan y Hargreaves, se definía a los docentes como artesanos. Esa idea del docente como artesano, acostumbrado a hacer sus propios diseños, con sus propios medios y con su técnica desarrollada a partir de ensayo y error o bien de la observación de otros artesanos, casa poco con el uso de tecnologías en la enseñanza. Aunque en estos momentos hablar de tecnologías nos lleve inexcusablemente a pensar en computadores e Internet, no debemos olvidar que a lo largo de los últimos cincuenta años se han intentado introducir en las aulas otras tecnologías: desde la imprenta de Freinet, pasando por la televisión escolar, el proyector de cuerpos opaco, proyector de 
diapositivas, retroproyector, presentaciones audiovisuales, etc. Sin embargo, pocas de estas innovaciones tecnológicas han tenido un impacto profundo en el cambio de las prácticas tradicionales docentes, y no tenemos por qué pensar que esto va a cambiar.

Creo que existe una endémica desconfianza de los docentes ante las tecnologías y no creo que sea algo intencionado, sino quizás sea algo debido a que la presentación de las tecnologías, como productos acabados, ya diseñados y listos para utilizar, encaja muy mal con esa idea del docente como artesano que necesita despiezar los diseños y procesos para así poder apropiarse de ellos.

\section{La influencia incompleta de los docentes}

Con el devenir de los años, la evolución en nuestras sociedades y la implantación de los medios de comunicación masiva e Internet, se percibe cómo poco a poco la escuela y el trabajo de los docentes están dejando de configurar la principal fuente de influencia educadora en las nuevas generaciones. En definitiva, a la escuela y a los docentes les han salido competidores.

Estos competidores los encontramos claramente en la ingente y creciente cantidad de aprendizaje informal que los alumnos tienen paralelo al aprendizaje formal que proporciona la escuela. Tanto la televisión como Internet están representando una fuente de influencia de generación de modelos, patrones sociales y valores que compiten y a menudo se enfrentan con los que desde la escuela se promueven.
En muchos casos, los profesores perciben que las familias (cada vez más heterogéneas) dejan de ser los aliados privilegiados de la escuela y de los docentes. Éste es un fenómeno que está afectando claramente la identidad profesional docente, que está forzando a muchos profesores a reconfigurar su posición y compromiso con ciertos valores y a preocuparse más por esas otras influencias que amenazan con disminuir el posible impacto educativo que la escuela tradicionalmente ha tenido.

\section{Empezar a enseñar: cuanto más dificil mejor}

Un aspecto que caracteriza a la docencia es su falta de preocupación por la forma como se integran los docentes en la enseñanza. Ya en otro trabajo reciente he tenido la oportunidad de desarrollar esta idea (Marcelo, 2006). La inserción profesional en la enseñanza es el periodo de tiempo que abarca los primeros años, en los cuales los profesores han de realizar la transición desde estudiantes a docentes. Es un periodo de tensiones y aprendizajes intensivos en contextos generalmente desconocidos y durante el cual los profesores principiantes deben adquirir conocimiento profesional, además de conseguir mantener un cierto equilibrio personal. Es éste el concepto de inserción que asume Vonk (1996), autor holandés con una década de investigaciones centradas en éste ámbito: "definimos la inserción como la transición desde profesor en formación hasta llegar a ser un profesional autónomo. La inserción se puede entender mejor como una parte de un continuo en el proceso de desarrollo profesional de los profesores" (p. 115). 
Conviene insistir en esta idea de que el periodo de inserción es un periodo diferenciado en el camino de convertirse en profesor. No es un salto en el vacío entre la formación inicial y la formación continua sino que tiene un carácter distintivo y determinante para conseguir un desarrollo profesional coherente y evolutivo (Britton, Paine, Pimm, \& Raizen, 2002). El periodo de inserción y las actividades propias que le acompañan varían mucho entre los países. En algunos casos se reducen a actividades burocráticas y formales. En otros casos, como veremos más adelante, configuran toda una propuesta de programa de formación cuya intención es asegurar que los profesores entren en la enseñanza acompañados por otros que pueden ayudarle.

Los profesores principiantes tienen, según Feiman-Nemser (2001), dos tareas que cumplir: deben enseñar y deben aprender a enseñar. Independientemente de la calidad del programa de formación inicial que hayan cursado, hay algunas cosas que sólo se aprenden en la práctica y ello repercute en que este primer año sea un año de supervivencia, descubrimiento, adaptación, aprendizaje y transición. Las principales tareas con que se enfrentan los profesores principiantes son: adquirir conocimientos sobre los estudiantes, el currículo y el contexto escolar; diseñar adecuadamente el currículo y la enseñanza; comenzar a desarrollar un repertorio docente que les permita sobrevivir como profesores; crear una comunidad de aprendizaje en el aula y continuar desarrollando una identidad profesional. El problema es que esto deben hacerlo, en general, cargados con las mismas responsabilidades que los profesores más experimentados (Marcelo, 1999).
Pero la realidad cotidiana del profesorado principiante nos indica que muchos profesores abandonan y lo hacen por estar insatisfechos con su trabajo, debido a los bajos salarios, a los problemas de disciplina con los alumnos, a la falta de apoyo y a las pocas oportunidades para participar en la toma de decisiones. Decía Cochran-Smith (2004) que "para permanecer en la enseñanza, hoy y mañana, los profesores necesitan condiciones en la escuela que les apoyen y les creen oportunidades para trabajar con otros educadores en comunidades de aprendizaje profesional en lugar de hacerlo de forma aislada" (p. 391). Ahondando en las causas, la National Commission on Teaching and America's Future (1996), citado por Horn, Sterling, \& Subhan (2002), estableció cinco razones por las cuales los profesores abandonan la docencia:

- Porque se les asigna la enseñanza de los alumnos con mayores dificultades.

- Porque se les inunda con actividades extracurriculares.

- Porque se les pone a enseñar en una especialidad o nivel diferente al que posee.

- Porque no reciben apoyo desde la administración.

- Porque se sienten aislados de sus compañeros.

\section{Los desafíos}

He identificado catorce constantes. Quizás podrían ser más. Quizás podrían agruparse. Pero son lo suficientemente sugerentes como para desarrollar el debate que espero propicie esta 
intervención. Como comentaba al comienzo de esta ponencia, la profesión docente se encuentra en una encrucijada que debe resolver dando respuestas innovadoras a los problemas que la educación tiene planteados en nuestros días. Para nada vale recordar aquello de que "cualquier tiempo pasado fue mejor". El tiempo que nos ha tocado vivir es éste y en él nos jugamos algo tan importante como es la capacidad de permanencia de una institución que, a lo largo de los últimos siglos, ha contribuido como ninguna otra a asegurar la igualdad y el acceso al conocimiento de las personas. No creo que sea muy exagerada esta afirmación, sobre todo cuando en determinados países se diseñan políticas educativas que, bajo el paraguas de la libertad de elección y la defensa de la competencia, ocultan intenciones de control y privatización de la educación. El desafío, por lo tanto, es transformar la profesión docente en una profesión del conocimiento. Una profesión que sea capaz de aprovechar las oportunidades de nuestra sociedad para conseguir que respete uno de los derechos fundamentales: el derecho de todos los alumnos y alumnas, de adultos y adultas, de aprender.

\section{Referencias}

Beijaard, D., Meijer, P. \& Verloop, N. (2004). Reconsidering Research on Teachers' Professional Identity. Teaching and Teacher Education, 20, 107-128.

Bird, T. \& Little, J. W. (1986). How schools organize the teaching occupation. The Elementary School Journal, 86 (4), 493-512.
Blumenfeld, P., C., Marx, R., W., Patrick, H., Krajcik, J. y Soloway, E. (1998). Teaching for Understanding. In B. J. Biddle, T. L. Good \& I. Goodson (Eds.), International Handbook of Teachers and Teaching (pp. 819-878). (Vol. 2). London, UK: Kluwer Academic Publishers.

Bolívar, A. (2006). La identidad profesional del profesorado de secundaria: crisis y reconstrucción. Málaga, España: Aljibe.

Bonk, C. \& Cunningham, D. (1998). Searching for Learner-Centered, Constructivism, and Sociocultural Components of Collaborative Educational Learning Tools. In C. Bonk \& K. King (Eds.), Electronic collaborators (pp. 25-50). New Jersey, USA: Lawrence Erlbaum Ass.

Borko, H. \& Putnam, R. T. (1996). Learning to teach. In D. C. Berliner \& R. C. Calfee (Eds.), Handbook of Educational Psychology (pp. 673-708). New York, USA: Macmillan.

Britton, E., Paine, L., Pimm, D., \& Raizen, S. (2002). Comprehensive teacher induction. Dordrecht, Netherlands: Kluwer Academic Press.

Buchmann, M. (1984). The Priority of Knowledge and Understanding in Teaching. In L. Katz \& J. Raths (Eds.), Advances in teacher education (Vol. 29-50). Norwood: Ablex. 
Bullough, R. (1998). Becoming a Teacher: Self and the Social Location of Teacher Education. In B. J. Biddle, T. L. Good \& I. Goodson (Ed.), International Handbook of Teacher and Teaching (pp. 79-134). London, UK: Kluwer.

Chapman, J., \& Aspin, D. (2001). Schools and the Learning Community: Laying the Basis for Learning Across the Lifespan. In D. Aspin, J. Chapman, M. Hatton \& Y. Sawano (Eds.), International Handbook of Lifelong Learning (pp. 405-446). (Vol. 2). London, UK: Kluwer Academic Publishers.

Cochran-Smith, M. (2004). Stayers, leavers, lovers, and dreamers. Insight about teacher retention. Journal of Teacher Education, 55 (5), 387-392.

Cochran-Smith, M. \& Fries, K. (2005). The AERA Panel on Research and Teacher Education: Context and Goals. In M. Cochran-Smith \& K. Zeichner (Eds.), Studying Teacher Education. The Report of the AERA Panel on Research and Teacher Education (pp. 37-68). New Jersey, USA: Lawrence Erlbaum Associates.

Cochran-Smith, M. \& Lytle, S. (1999). The Teacher Research Movement: A Decade Later. Educational Researcher, 28 (7), 15-25.
Darling-Hammond, L. (2000). Teacher Quality and Student Achievement: A Review of State Policy Evidence. Educational Policy Analysis Archives, 8 (1).

Darling-Hammond, L. (2001). El derecho de aprender. Crear buenas escuelas para todos. Barcelona, España: Ariel.

Day, C. (2001). Innovative Teachers: Promoting Lifelong Learning for All. In J. C. D. Aspin, M. Hatton and Y. Sawano (Eds.), International Handbook of Lifelong Learning (pp. 473-500). (Vol. 2). London, UK: Kluwer.

Day, C., Elliot, B., \& Kington, A. (2005). Reform, Standards and Teacher Identity: Challenges of Sustaining Commitment. Teaching and Teacher Education, 21, 563-577.

Dewey, J. (1938). Democracy and education: an introduction to the philosophy of education. New York, USA: WLC Books.

Duffy, T., Dueber, B. \& Hawley, C. (1998). Critical thinking in a distributed environment: A Pedagogical base for the design of conferencing systems. In C. Bonk \& K. King (Eds.), Electronic Collaborators (pp. 51-78). New Jersey, USA: Lawrence Erlbaum Ass.

Feiman-Nemser, S. (2001). From Preparation to Practice: Designing a Continuum to Strengthen and Sustain Teaching. Teachers College Record, 103 (6), 1013-1055. 
Fullan, M. \& Hargreaves, A. (1992). What's worth fighting for in your school? Working together for improvement. London, UK: Open University.

Gess-Newsome, J. (2003). Secondary Teachers' Knowledge and Beliefs about Subject Matter and their impact on instruction. In J. Gess-Newsome (Ed.), Examining pedagogical content knowledge. The construct and the its implication for science education (pp. 51-94). New York, USA: Kluwer Academic Publisher.

Grant, C. A. \& Wieczorek, K (2000). Teacher Education and Knowledge in "the Knowledge Society". The Need for Social moorings in Our Multicultural Schools. Teacher College Record, 102 (5), 913-935.

Hager, P. (2001). Lifelong Learning and the Contribution of Informal Learning. In D. Aspin, J. Chapman, M. Hatton \& Y. Sawano (Eds.), International Handbook of Lifelong Learning (pp. 79-92). London, UK: Kluwer.

Hargreaves, D. (1997). Road to the Learning Society. School Leaderchip and Management, 17 (1), 9-21.

Hargreaves, D. (1999). The knowledge-creating school. British Journal of Educational Studies, 47 (2), 122-144.
Hargreaves, A. \& Goodson, I. (1996). Teachers' professional lives: aspirations and actualities. London, UK: Falmer Press.

Himanen, P. (2001). The Hacker Ethic. New York, USA: Random House.

Horn, P., Sterling, H. \& Subhan, S. (2002). Accountability through "Best Practice" Induction Model. ERIC ED, 464039.

Hoyle, E. \& John, P. (1995). Professional knowledge and professional practice. London, UK: Cassell.

Huberman, M. (1993). The model of the independent artisan. In J. W. Little \& M. W. McLaughlin (Eds.), Teachers'Professional Relations in teachers' work. Individual, coIleagues and contexts (pp. 11-50). Chicago, USA: Teacher College Press.

Kagan, D. (1992). Professional Growth Among Preservice and Beginning Teachers. Review of Educational Research, 62 (2), 129-169.

Labaree, D. (1998). Educational Researchers: Livingwith a Lesser Form of Knowledge. Educational Researcher, 27 (8), 4-12.

Ladson-Billing, G. (1998). Preparing Teachers for Diversity. In L. Darling-Hammond \& G. Sykes (Eds.), Teaching as a Learning Pro- 
fessional. Handbook of Policy and Practice (pp. 86-121). San Francisco, California, USA: Jossey-Bass.

Lasky, S. (2005). A Sociocultural Approach to Understanding Teacher Identity, Agency and Professional Vulnerability in a Context of Secondary School Reform. Teaching and Teacher Education, 21, 899-916.

Little, J. W. \& McLaughlin, M. W. (1993). Perspectives on Cultures and Contexts of Teaching. In J. W. Little \& M. W. McLaughlin (Eds.), Teachers' work, individuals, colleagues, and contexts (pp. 1-8). New York. USA: Teacher College Press.

López, J. y Sánchez, M. (2000). Acerca del cambio en los sistemas complejos. En A. Estebaranz (Ed.), Construyendo el cambio: perspectivas y propuestas de innovación educativa. Sevilla, España: Secretariado de Publicaciones de la Universidad.

Lortie, D. (1975). Schoolteachers: a sociological study. Chicago, USA: University of Chicago Press.

Magnusson, S., Krajcik, J., \& Borko, H. (2003). Nature, Sources, and Development of Pedagogical Content Knowledge for Science Teaching. In J. Gess-Newsome (Ed.), Examining pedagogical content knowledge. The construct and its implication for science education (pp. 95-132). New York, USA: Kluwer Academic Publisher.

Marcelo, C. (1996). Constantes y desafíos actuales de la profesión docente. Revista de Educación, (306), 205-243.

Marcelo, C. (1998). Formación de profesores para el cambio educativo. Barcelona, España: EUB.

Marcelo, C. (1999). Estudio sobre estrategias de inserción profesional en Europa. Revista Iberoamericana de Educación, (19), 101-144.

Marcelo, C. (2002). Aprender a enseñar para la sociedad del conocimiento. Educational Policy Analysis Archives, 10 (35).

Marcelo, C. (2006). Políticas de inserción a la docencia: de eslabón perdido a puente para el desarrollo profesional docente. Informe elaborado para el Programa para la Reforma Educativa en América Latina y presentado el 23 de noviembre en el Seminario "Política para integrar a los nuevos profesores en la profesión docente".

Onofre, M. (2000). Conhecimento prático, autoeficácia e qualidade do ensino. Um Estudo Multicaso em Professores de Educaçao Física. Lisboa, Portugal: Universidade Tecnica de Lisboa. 
Organización para la Cooperación y el Desarrollo Económico, OCDE. (2005). Teachers matter: attracting, developing and retaining effective teachers. París, Francia: OCDE.

Pajares, M. F. (1992). Teachers' Beliefs and Educational Research: Cleanning Up a Messy Construct. Review of Educational Research, 62 (3), 307-332.

Richardson, V. (1996). The Role of Attitudes and Beliefs in Learning to Teach. In W. R. Houston, M. Haberman \& J. Sikula (Eds.), Handbook of Research on Teacher Education (pp. 102-119). New York, USA: Macmillan.

Schön, D. (1983). The reflective practitioner. New York, USA: Basic Books.

Shulman, L. (1992). Renewing the Pedagogy of Teacher Education: The Impact of Subject Specific Conceptions of Teaching. Paper presented at the Simposium about "Didácticas Específicas en la Formación de Profesores", Santiago de Compostela.

Shulman, L. (1998). Theory, Practice, and the Education of Professional. The Elementary School Journal, 98 (5), 511-526.

Sloan, K. (2006). Teacher Identity and Agency in School Worlds: Beyond the all-good/allbad Discourse on Accountability-explicit
Curriculum Policies. Curriculum Inquiry, 36 (2), 119-152.

Smyth, J. (1995). Teacher' Work and the Labor Process of Teaching. In T. R. Guskey \& M. Huberman (Eds.), Professional development in education: new paradigms and practices. (pp. 69-91). New York: Teacher College Press.

Sykes, G. (1999). Teacher and Student Learning. Stregthening their Connection. In L. Darling-Hammond \& G. Sykes (Eds.), Teaching as a learning profession. Handbook of policy and practice (pp. 151-179). San Francisco, California, USA: Jossey-Bass.

Talbert, J. \& McLaughlin, M. (2002). Professional Communities and the Artisan Model of Teaching. Teacher and teaching, 8 (3), $325-343$.

Tardif, M. (2004). Los saberes del docente y su desarrollo profesional. Madrid, España: Narcea.

Terigi, F. (2007). Desarrollo profesional continuo y carrera docente en América Latina. Ponencia presentada en el Encuentro Internacional sobre Desarrollo Profesional Docente en América Latina, organizado por la PREAL. 
Tomlinson, H. (1997). Continuing Professional Development in the Profession. In $\mathrm{H}$. Tomlinson (Ed.), Managing continuing professional development in schools (pp. 13-26). London, UK: Paul Chapman.

Van Veen, K., Sleegers, P. \& Van de Ven, P. (2005). One teacher's identity, emotions, and commitmet to change: a case study into the cognitive-affective processes of a secondary school teacher in the context of reforms. Teaching and Teacher Education, 21, 917-934.

Vonk, J. H. C. (1996). A Knowledge Base for Mentors of Beginning Teachers: Results of a Dutch Experience. In R. McBridge (Ed.), Teacher Education Policy (pp. 112-134). London, UK: Falmer Press.
Wideen, M., Mayer-Smith, J. \& Moon, B. (1998). A Critical Analysis of the Research on Learning to Teach: Making the Case for an Ecological Perspective on Inquiry. Review of Educational Reseach, 68, 130-178.

Wilson, B. (1996). What is a constructivist learning environment? In B. Wilson (Ed.), Constructivist learning environments: case studies in instructional design (pp. 3-7). Englewood Cliffs: Educational Technology Publications.

Yinger, R. \& Hendricks, M. (2000). The Language of Standards and Teacher Educatio Reform. Educational Policy, 14 (1), 94-106.

Zeichner, K. M. (1980). Research Subcontract with the National Center for Research in Teacher Education. Michigan, USA: Michigan State University. 